\title{
The effect of COVID-19 pandemic on hospitalizations and disease characteristics of adolescents with anorexia nervosa
}

\author{
Lotem Goldberg ${ }^{1,2} \cdot$ Adi Ziv $^{2,3} \cdot$ Yoav Vardi $^{1,2} \cdot$ Shai Hadas ${ }^{1,2} \cdot$ Tarek Zuabi $^{1,2} \cdot$ Lital Yeshareem $^{2,4} \cdot$ Tomer Gur $^{2,5}$. \\ Shelly Steinling ${ }^{1,2} \cdot$ Oded Scheuerman $^{1,2} \cdot$ Yoel Levinsky $^{1,2}$ (I)
}

Received: 11 October 2021 / Revised: 11 December 2021 / Accepted: 13 December 2021 / Published online: 3 January 2022

(c) The Author(s), under exclusive licence to Springer-Verlag GmbH Germany, part of Springer Nature 2022

\begin{abstract}
Social distancing and quarantines have major negative psychological implications. Our aim was to study the rate of pediatric hospitalizations due to anorexia nervosa (AN) during the first year of coronavirus disease 2019 (COVID-19) pandemic as compared to previous years, with regard to clinical and laboratory parameters. This is a retrospective study in a tertiary pediatric hospital in Israel. Data regarding inpatient hospitalizations due to AN were retrieved, then epidemiological, clinical, and laboratory parameters compared. During the entire study period, 275 hospitalizations were due to AN: 94 patients were admitted during the 12 months of the pandemic as compared to a yearly mean of 45.25 during 2015-2019, resulting in a 2.4-fold increase $(p<0.001)$. The mean admission age and female predominance were similar in the two study groups. The weight of the patients at admission was higher during the COVID-19 period (44.5 kg vs. $41.2 \mathrm{~kg}, p=0.004)$, and fewer patients had clinical signs typical of AN upon physical examination $(p=0.022)$. There was a $35 \%$ reduction in median hospitalization duration (9 days [IQR 8.21] in 2020-2021 and 14 [IQR 6, 16.75] days in 2015-2019, $p=0.01$ ). No other differences were found.

Conclusions: During the first year of the COVID-19 pandemic, there was a significant increase in the number of adolescents hospitalized with AN. Nevertheless, disease characteristics were not more severe as compared to the preceding 5 years.

\section{What is Known:}

- Social distancing and quarantines were announced during the COVID-19 pandemic in numerous countries. These measures have potential negative psychological effects on adolescents.

What is New:

- During the COVID-19 pandemic period, there was an increase in the number of hospitalizations of adolescents with AN, although disease characteristics were not more severe as compared to the preceding 5 years.
\end{abstract}

Keywords Anorexia nervosa $\cdot$ Eating disorder $\cdot$ COVID-19 $\cdot$ Pandemic

\section{Abbreviations}

AAN Atypical anorexia nervosa

AN Anorexia nervosa

COVID-19 Coronavirus disease 2019

ED Eating disorder

OCD Obsessive-compulsive disorder

Communicated by Peter de Winter

Drs. Oded Scheuerman and Yoel Levinsky contributed equally to this work.

Lotem Goldberg

lotemgo@clalit.org.il; lotem.yahav@gmail.com

Extended author information available on the last page of the article

\section{Introduction}

The coronavirus disease 2019 (COVID-19) pandemic led to strict infection prevention strategies including mandatory mask wearing, frequent hand sanitation, and social distancing. Moreover, in order to control the pandemic, many governments imposed nationwide quarantines, with closure of educational facilities and airports, and cancellation of social events. Social distancing and quarantines had major negative psychological implications [1].

Anorexia nervosa (AN) is one of the most common mental disorders in adolescents. AN is often associated with other mental health co-morbidities, such as mood or anxiety disorders, and obsessive-compulsive disorder (OCD) [2]. 
Thus, it can be expected that the negative implications of COVID-19 restrictions will be more noticeable in individuals with AN, in whom emotional and physical isolation are more common [3].

Lin et al. showed an increase in both outpatient visits and inpatient admissions (partial or full hospitalizations) of adolescents and young adults with eating disorders (EDs) who sought medical care during the pandemic [4]. Otto et al. reported a greater than 2-fold increase in pediatric hospitalizations due to AN during the pandemic, as compared to previous years [5]. In contrast, total hospitalizations for patients with all general medical conditions declined during the pandemic, as compared to those with EDs [6].

We aimed to compare the rate of pediatric medical hospitalizations due to AN during the COVID-19 pandemic, to previous years, in a tertiary pediatric medical center in Israel. Furthermore, we compared clinical and laboratory parameters upon admission.

\section{Materials and methods}

Schneider Children's Medical Center (SCMC) is the largest, tertiary pediatric hospital in Israel. Patients with EDs are usually treated ambulatory. Adolescents with EDs who seek emergent care usually undergo an initial assessment, after which the decision regarding hospitalization is made according to the criteria of the American Academy of Pediatrics [7], whereby the aim of hospitalization is to achieve physical stabilization in accordance with the above guidelines. A decision regarding discharge is made by a multidisciplinary team that includes a certified pediatrician, mental health expert, and nutritionist.

We retrieved information on all hospitalizations encoded with the diagnosis of AN between January 2015 and May 2021 from the hospital's computerized system. Certified physicians reviewed each medical file to confirm the diagnosis and collect relevant medical information. The number of hospitalizations was examined for each quarter, and epidemiological and clinical data collected for each hospitalization.

\section{Definitions and timeframe}

We used the Centers for Disease Control and Prevention (CDC) data for calculating $z$-scores for BMI according to age and sex. Since there is no official definition of atypical anorexia nervosa (AAN) in children, we used the definition suggested by Garber et al.: $>85 \%$ of the median BMI standardized to age and sex [8].

In March 14, 2020, Israel educational institutions were closed due to the spread of the pandemic. In March 25, 2020, a general lockdown was declared, whose restrictions were gradually reduced in the beginning of May 2020. We assumed that the implications of social measures on ED would take a few weeks to develop. In addition, it could be argued that some patients were afraid to seek help at the emergency department during the lockdown. Accordingly, we chose May 1, 2020, as the relevant date for examining the effects of the social distancing measures on AN hospitalizations and disease characteristics.

\section{Statistical analysis}

Comparison of general characteristics at admission between study periods was conducted using frequencies, means, and SDs. Independent $t$-tests for continuous variables, and chi-square tests for categorical variables, were used to examine differences between the two groups with regard to demographic and clinical characteristics (e.g., age, gender, BMI, menses, duration of AN, purging behavior, and lab results). Continuous variables with non-normal distributions were compared using the nonparametric Mann-Whitney $U$ test. A number of quarterly AN hospitalizations between periods were compared using chi-square test. Binary logistic regression was used to compare associations between the study periods and independent variables. IBM® SPSS software version 25 for Windows (IBM Corp, Armonk, New York, USA) and the Table 1 package in $\mathrm{R}$ were used for data analysis [9].

\section{Results}

During the study period, there were 275 hospitalizations due to AN diagnosis in the three general pediatric wards (94 in the 12-month COVID-19 period of 2020-2021 as compared to a yearly mean of 45.25 in $2015-2019$, a 2.4fold increase $(p<0.001)$ (Fig. 1).

The mean admission age was similar between the two study groups, as well as female predominance $(83 \%$ and $88.4 \%$, respectively, $p=0.288$ ) and previous psychiatric comorbidities $(6.4 \%$ and $7.2 \%, p=1.0)$.

Regarding disease characteristics, the weights of the patients at admission were higher during the COVID19 period (44.5 kg vs. $41.2 \mathrm{~kg}$, respectively, $p=0.004$ ) although the corrected BMIs and BMI percentiles were similar ( $Z$ score of $-1.4 \pm 1.2$ vs. $-1.6 \pm 1.4, p=0.3669$ ). Disease duration at the time of admission was also similar between two study groups $(9.17 \pm 6.88$ vs. $10.49 \pm 9.80$, respectively, $p=0.383$ ).

During the physical examination, fewer patients showed clinical signs typical for AN $(p=0.022)$. Prevalence of AAN was similar among the two groups. Other clinical parameters 
Table 1 Epidemiological and clinical characteristics of adolescents with anorexia nervosa admitted to the general pediatrics wards during the COVID-19 pandemic as compared to previous years

\begin{tabular}{|c|c|c|c|}
\hline Parameter & $\begin{array}{l}\text { COVID-19 period (2020- } \\
2021), n(\%)\end{array}$ & $\begin{array}{l}\text { Previous years (2015- } \\
2019), n(\%)\end{array}$ & $P$-value \\
\hline Number & 94 & 181 & NA \\
\hline Gender $(F)$ & $78(83.0)$ & $160(88.4)$ & 0.288 \\
\hline Admission age, mean \pm SD & $14.63 \pm 1.54$ & $14.68 \pm 2.07$ & 0.854 \\
\hline Weight at admission $(\mathrm{kg})$, mean $\pm \mathrm{SD}$ & $44.57 \pm 9.21$ & $41.23 \pm 8.90$ & 0.004 \\
\hline Standardized BMI ( $z$ score $)$ & $-1.4 \pm 1.2$ & $-1.6 \pm 1.4$ & 0.3669 \\
\hline Atypical anorexia nervosa (AAN) & $21(24)$ & $41(23)$ & 1.0 \\
\hline Disease duration (months), mean $\pm \mathrm{SD}$ & $9.17 \pm 6.88$ & $10.49 \pm 9.80$ & 0.383 \\
\hline Psychiatric comorbidities at admission & $6(6.4)$ & $13(7.2)$ & 1.0 \\
\hline \multicolumn{4}{|l|}{ Menses: } \\
\hline Intact & $23(59.0)$ & $73(61.3)$ & \multirow[t]{2}{*}{0.204} \\
\hline Amenorrhea & $12(30.8)$ & $23(19.3)$ & \\
\hline Any clinical sign of $\mathrm{AN}$ in physical examination, mean $\pm \mathrm{SD}$ & $16(20.5)$ & $51(36.4)$ & 0.022 \\
\hline Purging behaviors, mean \pm SD & $10(16.7)$ & $23(16.0)$ & 1.0 \\
\hline Hospitalization days, median (IQR) & $9.00(6.00,16.75)$ & $14.00(8.00,21.00)$ & 0.01 \\
\hline Nasogastric tube feeding during hospitalization, mean \pm SD & $38(48.7)$ & $88(59.9)$ & 0.144 \\
\hline Any psychiatric medication during hospitalization, mean \pm SD & $40(43.0)$ & $67(39.0)$ & 0.609 \\
\hline Minimal heart rate $(\mathrm{BPM})$, mean \pm SD & $44.33 \pm 9.74$ & $45.01 \pm 9.88$ & 0.594 \\
\hline White blood cell count at admission $(\mathrm{K} / \mathrm{micl})$, mean $\pm \mathrm{SD}$ & $4.97 \pm 1.51$ & $5.48 \pm 1.68$ & 0.031 \\
\hline Triiodothyronine (T3) level (pmol/L), mean \pm SD & $3.64 \pm 0.92$ & $3.43 \pm 0.83$ & 0.193 \\
\hline Minimal phosphate level $(\mathrm{mg} / \mathrm{dl})$ during hospitalization, mean $\pm \mathrm{SD}$ & $3.77 \pm 0.56$ & $3.73 \pm 0.61$ & 0.635 \\
\hline
\end{tabular}

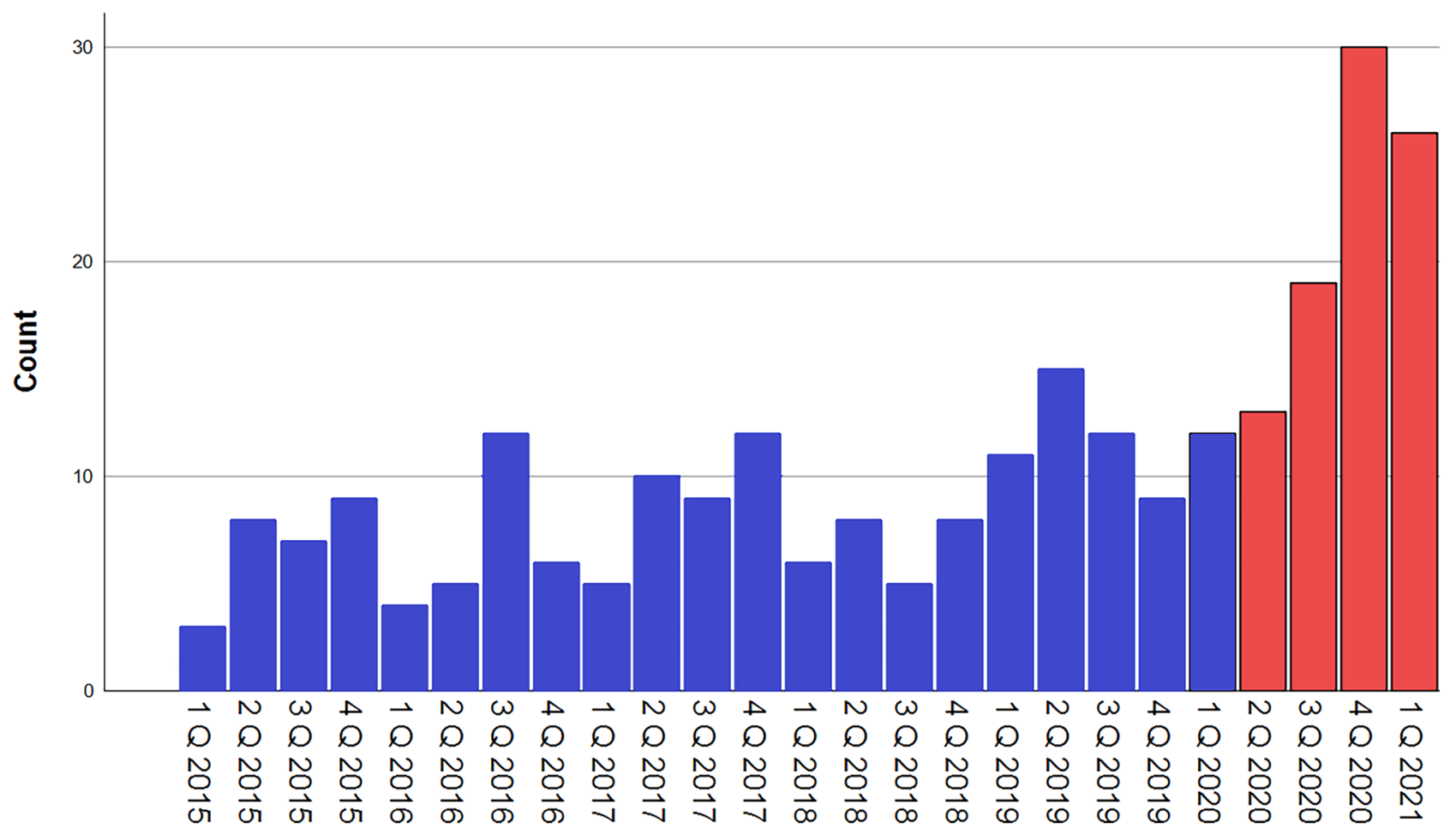

Fig. 1 Number of hospitalizations of adolescents with eating disorders in the general pediatric wards during 2015-2021. The COVID-19 pandemic period is marked 
(amenorrhea, purging behavior, minimal heart rate) were not significantly different. No differences were reported in baseline laboratory test evaluations between the two groups, except for white blood cell count, which was lower in the COVID-19 group, but still within normal range.

In terms of hospitalization course, there was a $35 \%$ reduction in median hospitalization duration (9 days [IQR 8.21] in 2020-2021, and 14 [IQR 6, 16.75] days in 2015-2019. $p=$ 0.01 ), but no significant differences in need for nasogastric (NG) tube feeding or prescriptions for psychiatric medications during hospitalization.

\section{Discussion}

In the present study, we found a significant increase in hospitalizations due to AN among adolescents during the COVID19 pandemic period in Israel, despite a drop in total pediatric admissions. In addition, patients during the COVID-19 period presented with fewer physical findings related to AN and higher weights at admission, and had shorter lengths of hospitalization. No other differences in clinical features examined were found.

During the pandemic, social distancing measures and closures were adopted globally, while concerns were expressed about the possible impact on the mental health of children and adolescents. With time, reports of an increase in incidence of mental and psychiatric disorders appeared. Specifically, Spettigue et al. reported that many adolescents with EDs who identified the pandemic as a trigger for their EDs had a greater tendency to become medically unstable and require hospitalization [10]. Subsequently, there were reports of an increase in mental health related emergency department visits as well as an increase in the numbers of adolescents hospitalized with EDs in the USA [5] and Australia [11].

At the beginning of the pandemic, some hoped that social distance measures would reduce levels of anxiety and thus the incidence of EDs [12]. Unfortunately, our research shows an increase in the incidence of these diseases during this period.

It is important to note that during the first months of the pandemic, there was a decline in total hospitalizations in our institution, among others, probably due to the pandemic restrictions [6], which further supports the significance of the increase in number of cases of AN during that period.

To the best of our knowledge, this is the first study to examine both the prevalence and clinical characteristics of hospitalizations due to AN during the pandemic period. Clinical differences were found ostensibly "to favor" the COVID-19 period, with fewer physical findings among inpatients and a shorter duration of hospitalization. We suggest that our findings indicate that although the prevalence of hospitalizations increased, the medical state at presentation was not worse, perhaps due to the fact that parents could monitor their child's behaviors more closely during the pandemic.

It is also possible that many young people who would not normally develop an ED, but experienced a restrained lifestyle which included changes in food availability, fewer outdoor activities, and over-exposure to social media had increased anxiety, which eventually developed into an ED and later became medically unstable. We assume that the shorter duration of hospitalization was influenced by both medical staff and families' supporting discharge as soon as possible, which might have led to increased motivation to adhere to the ED treatment protocol.

Our study has limitations, mainly due to its retrospective nature. In addition, it can be argued there were changes in the clinical management and decision making during this period, which might have influenced the duration of hospitalization, but it should be emphasized there were no changes in treatment protocols during these periods.

In conclusion, during the COVID-19 pandemic period, there was an increase in the number of hospitalizations of adolescents with AN, although disease characteristics were not more severe as compared to the preceding 5 years. These findings should raise awareness of the mental difficulties experienced by children and adolescents during the COVID-19 pandemic.

Authors' contributions Drs. Goldberg and Ziv conceptualized and designed the study, drafted the initial manuscript, and reviewed and revised the manuscript. Drs. Levinsky and Scheuerman conceptualized and designed the study, and reviewed and revised the manuscript. Drs. Hadas, Vardi, Yesharim, Zuabi, Gur, and S. Steinling coordinated and supervised data collection, collected data, participated in data analysis, and reviewed and revised the manuscript. All authors approved the final manuscript as submitted and agree to be accountable for all aspects of the work.

Availability of data and material The data that support the findings of this study are available from the corresponding author (LG), upon reasonable request.

\section{Declarations}

Ethics approval The study was approved by the Research Ethics Board of Rabin Medical Center (approval no. RMC-20-0363).

Consent to participate Not applicable.

Consent for publication Not applicable.

Conflict of interest The authors declare no competing interests. 


\section{References}

1. Brooks SK, Webster RK, Smith LE, Woodland L, Wessely S, Greenberg N et al (2020) The psychological impact of quarantine and how to reduce it: rapid review of the evidence. Lancet 395:912-920. https://doi.org/10.1016/S0140-6736(20)30460-8

2. Herpertz-Dahlmann B (2015) Adolescent eating disorders: update on definitions, symptomatology, epidemiology, and comorbidity. Child Adolesc Psychiatr Clin N Am 24:177-196. https://doi.org/ 10.1016/j.chc.2014.08.003

3. Touyz S, Lacey H, Hay P (2020) Eating disorders in the time of COVID-19. J Eat Disord 8:19. https://doi.org/10.1186/ s40337-020-00295-3

4. Lin JA, Hartman-Munick SM, Kells MR, Milliren CE, Slater WA, Woods ER et al (2021) The impact of the COVID-19 pandemic on the number of adolescents/young adults seeking eating disorder-related care. J Adolesc Heal 69:660-663. https://doi.org/10. 1016/j.jadohealth.2021.05.019

5. Otto AK, Jary JM, Sturza J, Miller CA, Prohaska N, Bravender $\mathrm{T}$ et al (2021) Medical admissions among adolescents with eating disorders during the COVID-19 pandemic. Pediatrics 148:e2021052201. https://doi.org/10.1542/peds.2021-052201

6. Gavish R, Levinsky Y, Dizitzer Y, Bilavsky E, Livni G, Pirogovsky A et al (2021) The COVID-19 pandemic dramatically reduced admissions of children with and without chronic conditions to general paediatric wards. Acta Paediatr Int J Paediatr 110:22122217. https://doi.org/10.1111/apa.15792
7. Harrington BC, Jimerson M, Haxton C, Jimerson DC (2015) Initial evaluation, diagnosis, and treatment of anorexia nervosa and bulimia nervosa. Am Fam Physician 91:46-52

8. Garber AK, Cheng J, Accurso EC, Adams SH, Buckelew SM, Kapphahn CJ et al (2019) Weight loss and illness severity in adolescents with atypical anorexia nervosa. Pediatrics 144:e20192339. https://doi.org/10.1542/peds.2019-2339

9. Panos A, Mavridis D (2020) TableOne: an online web application and $\mathrm{R}$ package for summarising and visualising data. Evid Based Ment Health 23:127-30. https://doi.org/10.1136/ebmental-2020-300162

10. Spettigue W, Obeid N, Erbach M, Feder S, Finner N, Harrison ME et al (2021) The impact of COVID-19 on adolescents with eating disorders: a cohort study. J Eat Disord 9:65. https://doi.org/10. 1186/s40337-021-00419-3

11. Haripersad YV, Kannegiesser-Bailey M, Morton K, Skeldon S, Shipton N, Edwards K et al (2021) Outbreak of anorexia nervosa admissions during the COVID-19 pandemic. Arch Dis Child 106:e15. https://doi.org/10.1136/archdischild-2020-319868

12. Akgül S, Akdemir D, Nalbant K, Derman O, Ersöz Alan B, Tüzün $\mathrm{Z}$ et al (2021) The effects of the COVID-19 lockdown on adolescents with an eating disorder and identifying factors predicting disordered eating behaviour. Early Interv Psychiatry. https://doi. org/10.1111/eip.13193

Publisher's Note Springer Nature remains neutral with regard to jurisdictional claims in published maps and institutional affiliations.

\section{Authors and Affiliations}

\section{Lotem Goldberg ${ }^{1,2} \cdot$ Adi Ziv $^{2,3} \cdot$ Yoav Vardi $^{1,2} \cdot$ Shai Hadas $^{1,2} \cdot$ Tarek Zuabi $^{1,2} \cdot$ Lital Yeshareem $^{2,4} \cdot$ Tomer Gur $^{2,5}$. Shelly Steinling ${ }^{1,2} \cdot$ Oded Scheuerman $^{1,2} \cdot$ Yoel Levinsky ${ }^{1,2}$ iD}

Adi Ziv

ziv.adi1@gmail.com

Yoav Vardi

vardiyo@gmail.com

Shai Hadas

shaivn90@gmail.com

Tarek Zuabi

Zoabitarek5@gmail.com

Lital Yeshareem

lital88@yahoo.com

Tomer Gur

tomergur21@gmail.com

Shelly Steinling

shellyli@clalit.org.il

Oded Scheuerman

Odedshv@clalit.org.il
Yoel Levinsky

yoel.lvn@gmail.com

1 Department of Pediatrics B, Schneider Children's Medical Center of Israel, Petah Tiqva, Israel

2 Sackler Faculty of Medicine, Tel Aviv University, Tel Aviv, Israel

3 Day Hospitalization Department, Schneider Children's Medical Center of Israel, Petach Tikva, Israel

4 Department of Pediatrics A, Schneider Children's Medical Center of Israel, Petah Tiqva, Israel

5 Department of Pediatrics C, Schneider Children's Medical Center of Israel, Petah Tiqva, Israel 